\title{
Incidence of Travelers' Diarrhea among Adult Foreign Travelers in Thailand: A Prospective Study
}

\author{
Chollasap Sharma, ${ }^{1,2}$ Kittiyod Poovorawan, ${ }^{1 *}$ Watcharapong Piyaphanee, ${ }^{1}$ Ngamphol Soonthornworasiri, ${ }^{3}$ \\ Piyada Angsuwatcharakon, ${ }^{4}$ Weerapong Phumratanaprapin, ${ }^{1}$ Wattana Leowattana, ${ }^{1}$ and Polrat Wilairatana ${ }^{1}$ \\ ${ }^{1}$ Department of Clinical Tropical Medicine, Faculty of Tropical Medicine, Mahidol University, Bangkok, Thailand; ${ }^{2}$ Department of Disease Control, \\ Ministry of Public Health, Institute for Urban Disease Control and Prevention, Bangkok, Thailand; ${ }^{3}$ Department of Tropical Hygiene, Faculty of \\ Tropical Medicine, Mahidol University, Bangkok, Thailand; ${ }^{4}$ Queen Saovabha Memorial Institute, Faculty of Medicine, Chulalongkorn University, \\ Bangkok, Thailand
}

\begin{abstract}
Travelers' diarrhea(TD) is common among foreign travelers to Thailand. We performed a prospective cohort study to determine the TD incidence among foreign adult travelers to Thailand. We retrieved baseline demographic data, travel plans, and health history on enrolling individuals and collected follow-up questionnaires on days 7,14 , and 28 from the day of arrival. We analyzed data from 349 eligible participants. The mean participants' age was 32.3 years; $55.4 \%$ were men. Most of the participants had visited a travel clinic for vaccinations and counseling after arrival in Thailand. The cumulative incidences of the participants developing TD were 14.0\% (49/349), 23.5\% (82/349), and 33.0\% (115/349) at 7 , 14 , and 28 days, respectively. The median time to develop TD was 9 days (interquartile range 5-18 days) post-arrival. Of 115 participants with TD, 64.3\% (74/115) consulted a physician, 1.7\% (2/115) were hospitalized, and 11.3\% (13/115) had to change their travel plans. We identified young age, eating street food, and not routinely washing hands after using a toilet as risk factors significantly associated with the incidence of TD using the log-rank test in our survival analysis. Up to one-third of foreign travelers developed diarrhea during the first month, and some cases were severe. Although no highly effective TD prevention method exists, the practice of good personal hygiene and avoidance of food and drinks derived from unsanitary sources are still recommended to reduce the risk of travelers' TD.
\end{abstract}

\section{INTRODUCTION}

Foreign travel may pose medical risks to travelers' health, especially in resource-limited settings. ${ }^{1}$ According to the $\mathrm{CDC}$, the attack rate of travelers' diarrhea (TD) ranges from $30 \%$ to $70 \%$ depending on multiple factors. ${ }^{2}$ Thailand has experienced more than a 10 million rise in foreigner's visits from the year 2012. The number of overnight travelers in 2017 was 35.6 million. $^{3}$

Thailand has been reclassified as an intermediate risk country for TD after being considered a high-risk country. ${ }^{4,5} \mathrm{~A}$ decade ago, a prospective cohort study among European travelers who visited a clinic and traveled between 1 and 8 weeks around developing countries found a lower TD rate incidence in Thailand than in other regions and reported an incidence of $17.4 \%$ for Southeast Asia. ${ }^{6}$ Since then, few studies have determined TD incidences in the region. One study among foreign backpackers with a median stay in Thailand of 30 days found about one-third of the participants had TD. Interestingly, about half of the cases occurred during the first 5 days. ${ }^{7}$ An airport-based study in Chiang Mai and Phuket found the attack rate of TD was 16\% among Australians and New Zealanders, 8\% among Europeans, and $7 \%$ among North Americans. ${ }^{8}$ Another study conducted in the Suvarnabhumi Airport found an incidence rate of $32.1 \%$ per person month. ${ }^{9}$

Although pathogens are only found in about half of stool specimens tested, ${ }^{5,8}$ the most frequent etiology worldwide is enterotoxigenic Escherichia coli. ${ }^{5,10,11}$ Acute diarrhea among travelers to Southeast and Southern Asia is commonly caused by Campylobacter species, which have been found to be the causative factor of Guillain-Barre syndrome. ${ }^{12,13}$ In Thailand, Plesiomonas species, Vibrio species, Campylobacter species, and norovirus were the most frequently isolated pathogens

*Address correspondence to Kittiyod Poovorawan, Department of Clinical Tropical Medicine, Faculty of Tropical Medicine, Mahidol University, 420/6 Rachavithee Rd., Bangkok 10400, Thailand. E-mail: kittiyod.poo@mahidol.ac.th from TD patients. ${ }^{14}$ The mean time to develop TD is less than a week ${ }^{7,9}$; however, a study found a mean time of 16 days. ${ }^{6}$ Fever with TD occurs in about $15 \%$ of cases. ${ }^{15,16}$ The highest rate of travelers visiting physicians because of TD has been reported as $8.8 \%{ }^{7}$ The highest rate of travelers changing their travel plans because of TD has been said to be $20.6 \% .^{15}$ The rate of hospitalization because of TD ranges from $1.25 \%$ to $3.2 \% .^{7,9,15}$

Given the lack of updated data or prospective studies to define the incidence of TD in Thailand, we designed this prospective study to estimate the precise incidence of TD and also find updated risk factors associated with the incidence of TD.

\section{MATERIALS AND METHODS}

Study design. This is a two-site prospective cohort study among adult foreign travelers who arrived in Thailand within a week. Our investigator team at the Travel Clinic of the Hospital for Tropical Diseases and Queen Saovabha Memorial Institute's Travel Clinic invited all travelers to participate in the study. Most of these travelers visited these travel clinics for travel-related vaccinations and counseling. We excluded travelers who had diarrhea within 6 hours after arrival. Eligible participants were foreign travelers who agreed to reply to the questionnaires provided on the day of enrollment and at least one follow-up questionnaire on days 7,14 , or 28 post-arrival. We invited participants who developed TD during their trip to revisit the Hospital for Tropical Diseases at Mahidol University, where we would isolate the etiologic pathogen.

Ethics approval and participants' consent. The Ethical Committee of the Faculty of Tropical Medicine, Mahidol University, Thailand, approved the protocol (MUTM 2016-08701). We obtained written informed consents from all the participants.

Data collection. We collected data on demographic and personal history risk factors for TD, including age, gender, nationality, and medical problems. Among the risk factors associated with the diet, we collected data on street food, tap water, alcohol, raw food, fruits, herbs, leftover food, ice, seafood, 


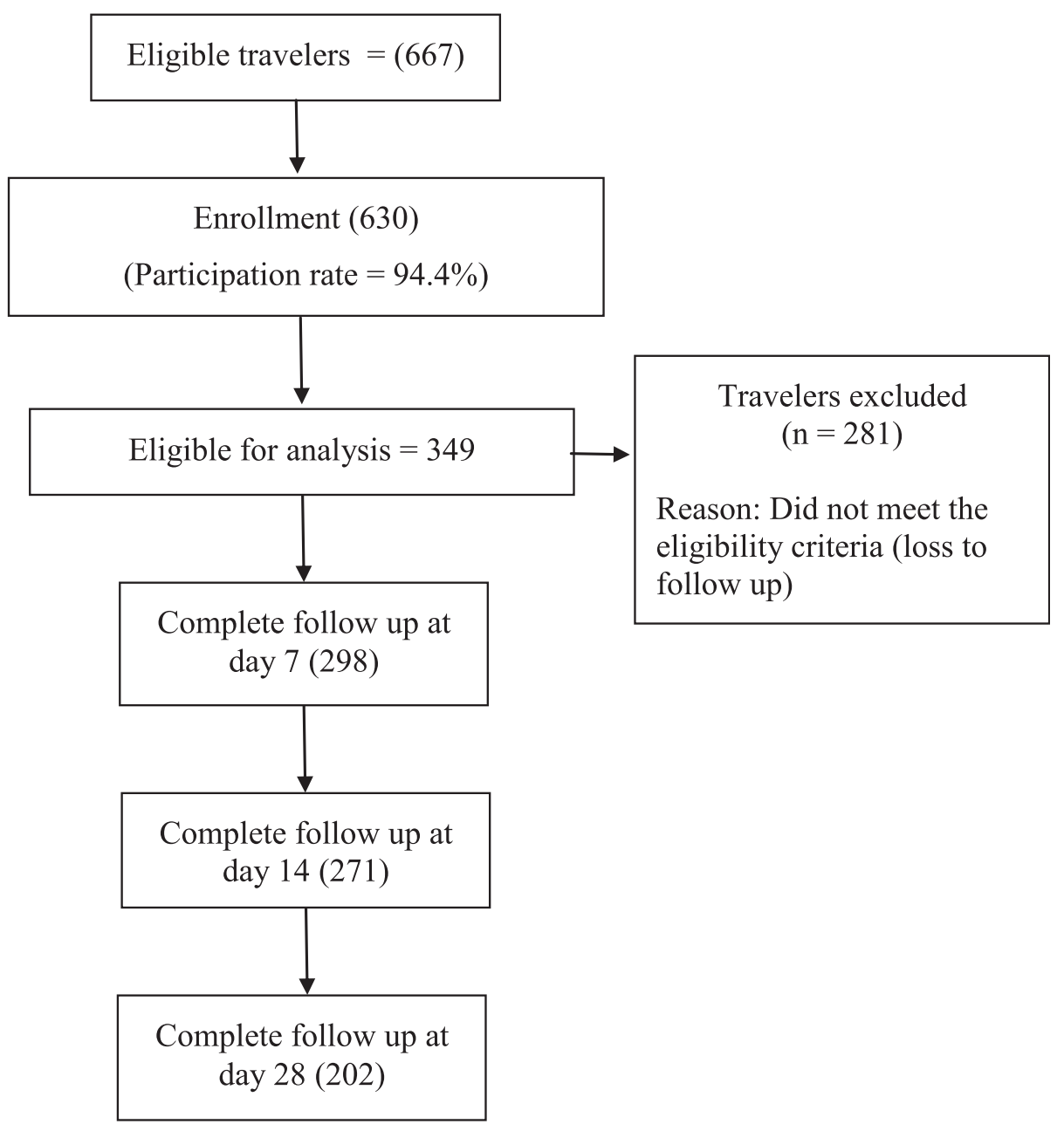

Figure 1. Flow diagram of the study.

vegetables, and insect ingestions. We also collected data on travel purposes, destination (city, countryside, and national parks), arrival from another Southeast Asian country, travel out of Thailand during the follow-up period, travel companion (solo, couple, with tour travel, and with children and elderly), and cruise trekking and swimming. Finally, we also obtained data on practices such as routinely washing hands before a meal, routinely washing hands after using the toilet, and consulting a physician about TD before the travel.
Questionnaires. The initial questionnaire enquired about demographic data, travel plans, and health history of the travelers. We followed up the participants using an online questionnaire through e-mail. We sent out the follow-up questionnaires to collect information about the first, second, and the fourth weeks after arrival in Thailand. From the follow-up questionnaires, we got detailed information about the travelers' diet, behavior, activities, and travel characteristics. We defined TD as the passage of three or more loose stools per day. ${ }^{6}$ In

TABLE 1

Comparison of demography among participants with and without diarrhea*

\begin{tabular}{|c|c|c|c|c|}
\hline Factors & All participants $(n=349)$ & With TD $(n=115)$ & Without TD $(n=234)$ & $P$-value \\
\hline Age (years), mean $\pm S D$ & $32.3 \pm 10.2$ & $30.5 \pm 8.4$ & $33.2 \pm 10.9$ & 0.010 \\
\hline Male gender, $n(\%)$ & $193(55.3)$ & $64(55.7)$ & $129(55.1)$ & 0.926 \\
\hline \multicolumn{5}{|l|}{ Nationality, $n(\%)$} \\
\hline European, North American, and Oceanian & $287(82.2)$ & $99(86.1)$ & $188(80.3)$ & \multirow[t]{2}{*}{0.181} \\
\hline Asian, South American, and African & $62(17.8)$ & $16(13.9)$ & $46(19.7)$ & \\
\hline \multicolumn{5}{|l|}{ Travel purpose, $n(\%)$} \\
\hline Tourism & $305(87.3)$ & 97 (84.3) & $208(88.8)$ & \multirow[t]{6}{*}{0.847} \\
\hline Visiting friends and relatives & 44 (12.6) & $18(15.6)$ & $26(11.1)$ & \\
\hline Volunteer & $22(6.3)$ & $8(6.9)$ & $14(5.9)$ & \\
\hline Business & $4(1.1)$ & $1(0.8)$ & $3(1.2)$ & \\
\hline Education & $15(4.2)$ & $4(3.4)$ & $11(4.7)$ & \\
\hline Other & $20(5.7)$ & $6(5.2)$ & $14(5.9)$ & \\
\hline
\end{tabular}

TD = travelers' diarrhea.

* The number in parenthesis is the column percentage of the total cases in each group. $P$-value compared the difference between participants with TD group and participants without TD group. 


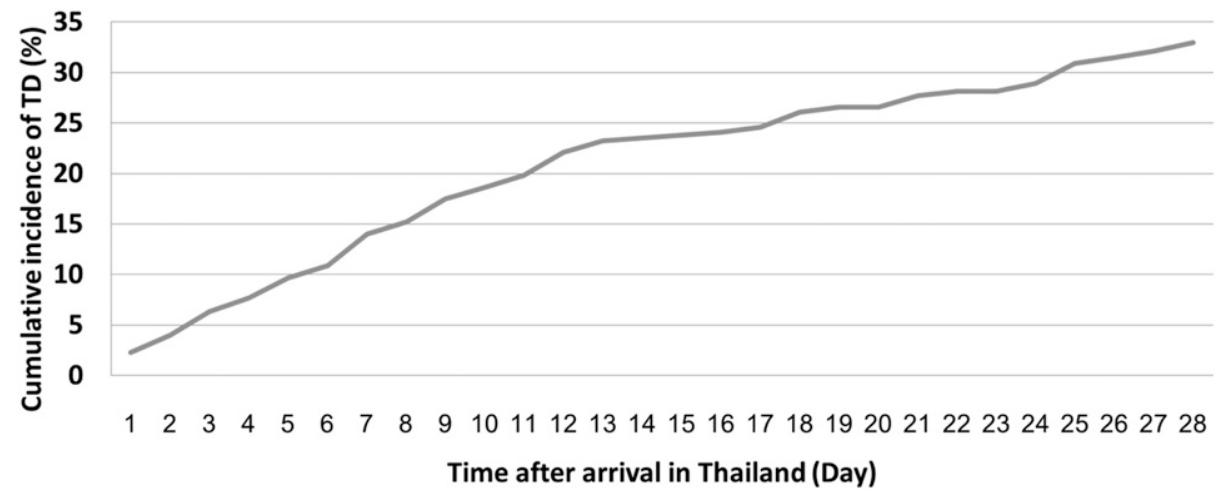

FIGURE 2. Cumulative incidence of travelers' diarrhea among adult international travelers in Thailand.

cases that were positive for TD, we collected information about symptoms and negative impacts on the travelers. Open-ended questions were used to enquire about diarrhea characteristics. In addition, we sent out a response e-mail to those affected to ask details about the illness. Please find the questionnaire used in the study in the Supplemental Questionnaire.

Statistical analysis. We conducted statistical analyses using the IBM SPSS Statistics for Windows, version 23 (IBM

TABLE 2

Independent factors associated with 28-day incidence of TD

\begin{tabular}{|c|c|c|c|}
\hline Factor (n) & Crude hazard ratio & $95 \% \mathrm{Cl}$ & $P$-value \\
\hline \multicolumn{4}{|l|}{ Demographic data } \\
\hline Age* $^{*}$ & 0.97 & $0.947-0.991$ & 0.006 \\
\hline Male gender & 0.96 & $0.716-1.496$ & 0.853 \\
\hline \multicolumn{4}{|l|}{ Nationality } \\
\hline African & 1 & - & - \\
\hline European & 0.81 & $-0.111-5.860$ & 0.832 \\
\hline North American & 0.96 & $0.132-6.919$ & 0.965 \\
\hline South American & 0.61 & $0.300-7.693$ & 0.605 \\
\hline Asian & 0.85 & $0.107-6.210$ & 0.845 \\
\hline Australian and Oceanian & 0.96 & $0.106-8.477$ & 0.961 \\
\hline Under university graduate & 1.36 & $0.879-2.113$ & 0.166 \\
\hline Atopy & 1.47 & $0.843-2.574$ & 0.176 \\
\hline Allergy & 1.48 & $0.953-2.284$ & 0.081 \\
\hline Consulted doctor about TD before travel & 1.42 & $0.975-2.078$ & 0.073 \\
\hline \multicolumn{4}{|l|}{ Diet and eating habit } \\
\hline Tap water & 1.62 & $1.001-2.634$ & 0.049 \\
\hline Alcohol & 1.11 & $0.715-1.727$ & 0.657 \\
\hline Raw food & 0.84 & $0.581-1.220$ & 0.357 \\
\hline Fruit & 0.73 & $0.389-1.401$ & 0.337 \\
\hline Vegetables & 0.98 & $0.632-1.515$ & 0.919 \\
\hline Herbal & 1.03 & $0.704-1.525$ & 0.870 \\
\hline Leftover food & 0.71 & $0.436-1.125$ & 0.181 \\
\hline Ice & 0.84 & $0.492-1.418$ & 0.504 \\
\hline Street food & 2.15 & $1.086-4.242$ & 0.028 \\
\hline Sea food & 0.88 & $0.607-1.273$ & 0.485 \\
\hline Insect & 0.72 & $0.320-1.641$ & 0.431 \\
\hline Bare hands & 1.11 & $0.762-1.601$ & 0.599 \\
\hline Wash hands before meal & 0.78 & $0.543-1.131$ & 0.182 \\
\hline Wash hands after using toilet & 0.67 & $0.452-0.983$ & 0.041 \\
\hline \multicolumn{4}{|l|}{ Activities of participants } \\
\hline Cruise travel & 0.99 & $0.507-1.596$ & 0.972 \\
\hline Swimming & 0.90 & $0.624-1.304$ & 0.584 \\
\hline Trekking & 1.13 & $0.760-1.664$ & 0.552 \\
\hline City travel & 1.36 & $0.547-27.97$ & 0.176 \\
\hline Countryside travel & 1.53 & $0.942-2.472$ & 0.086 \\
\hline National park travel & 1.37 & $0.941-2.003$ & 0.100 \\
\hline $\begin{array}{l}\text { Arrival from Southeast Asia or travel } \\
\text { abroad from Thailand }\end{array}$ & 1.54 & $1.022-2.311$ & 0.039 \\
\hline \multicolumn{4}{|l|}{ Traveling companion } \\
\hline Solo & 0.90 & $0.610-1.328$ & 0.586 \\
\hline With couple & 1.13 & $0.738-1.632$ & 0.508 \\
\hline With children or elderly & 0.39 & $0.160-0.961$ & 0.041 \\
\hline With group tour & 1.19 & $0.471-3.239$ & 0.734 \\
\hline With others & 1.23 & $0.796-1923$ & 0.353 \\
\hline
\end{tabular}

TD = travelers' diarrhea.

* Data represent continuous variables, for a one-unit increase in continuous predictor variable. 
TABLE 3

Significant factors in univariate survival and their result after the multivariate survival analysis

\begin{tabular}{|c|c|c|c|c|c|c|}
\hline Factors $(n)$ & Crude hazard ratio & $95 \% \mathrm{Cl}$ & $P$-value & Adjusted hazard ratio & $95 \% \mathrm{Cl}$ & $P$-value \\
\hline Age $^{\star}$ & 0.97 & $0.947-0.991$ & 0.006 & 0.97 & $0.952-0.996$ & 0.022 \\
\hline Tap water & 1.62 & $1.001-2.634$ & 0.049 & 1.85 & $1.128-3.022$ & 0.015 \\
\hline Street food & 2.15 & $1.086-4.242$ & 0.028 & 1.93 & $0.973-3.839$ & 0.060 \\
\hline With children or elderly & 0.39 & $0.160-0.961$ & 0.041 & 0.48 & $0.192-1.181$ & 0.109 \\
\hline Washing hands after using toilet & 0.67 & $0.452-0.983$ & 0.041 & 0.70 & $0.475-1.035$ & 0.074 \\
\hline $\begin{array}{l}\text { Arrival from Southeast Asia } \\
\quad \text { or travel abroad }\end{array}$ & 1.54 & $1.022-2.311$ & 0.039 & 1.60 & $1.058-2.411$ & 0.026 \\
\hline
\end{tabular}

Corp., Armonk, NY). We presented continuous data as means with SD (for normally distributed data) or as medians with range (for non-normally distributed data). Categorical data are presented as numbers and percentages.

We used survival analysis to find the associations between different factors and survival times to TD. We showed the cumulative survival probability of participants without TD using a Kaplan-Meier plot, and the log-rank test to compare survival curves between two or more groups of participants. Also, we used Cox-regression models to identify risk factors associated with the TD incidence. $P$-values $<0.05$ were regarded as statistically significant.

\section{RESULTS}

We collected data from December 2016 to May 2017 from adult international travelers who arrived in Thailand. All the participants were enrolled in the study within a week of their arrival; we screened 667 participants who were interested to join the research and enrolled 630 participants according to inclusion and exclusion criteria (Figure 1).

The age of the eligible participants ranged from 18 to 72 years (Table 1$)$. The mean age $\pm S D$ was $32.3 \pm 10.2$ years. We enrolled 227 North Americans, 218 Europeans, 150 Asians, 18 Australians and Oceanians, 10 South Americans, and seven Africans. Most of the travelers had a high level of education and were well prepared to manage TD.

The most common travel reason was tourism in 305 (87.4\%), followed by visiting friends and relatives (VFRs) in 44 (12.6\%), volunteering in $22(6.3 \%)$, business in four $(1.3 \%)$, education in 15 (4.3\%), and others in 20 (5.7\%). Some participants had more than one reason for their travel, especially among volunteers and VFRs. Travel to a city was the most common planned travel destination with 338 cases (96.9\%), followed by countryside visits in 266 cases (76.2\%), and to national parks in 196 cases $(56.2 \%)$. Of all the responders, $72(20.6 \%)$ traveled to Thailand from at least one Southeast Asian country. Most of them (181 or $52.0 \%)$ traveled as a couple, whereas 124 (35.5\%) traveled alone, $35(10.0 \%)$ with children or elderly, $15(4.3 \%)$ were part of a group tour, and $33(9.5 \%)$ had other companions.

Among the responders, 110 (31.5\%) consulted a physician about TD before their trip, and 235 (67.3\%) carried standby medication for diarrhea (155 or $44.4 \%$ carried antidiarrheal agents and 80 or $22.9 \%$ standby antibiotics). We found 13 (3.7\%) participants with TD on the day of enrollment.

Incidence and characteristics of TD. Of 349 responders, $14.0 \%$ (49/349), 23.5\% (82/349), and 33.0\% (115/349) developed TD during the first 7,14 , or 28 days after arrival, respectively (Figure 2).

Among 115 participants with TD, 74 (64.3\%) visited a physician, $13(11.3 \%)$ needed to change their travel plans, and two
$(1.7 \%)$ were hospitalized because of diarrhea. Among other symptoms of TD, 29 of 115 infected individuals (25.2\%) developed accompanying fever.

Among 66 participants who had experienced TD, the median duration of diarrhea was 2 days, with an interquartile range (IQR) from 1 to 3.5 days. Travelers who developed diarrhea visited many tourist destinations, including Bangkok Province, Chiangmai Province, Pai District, Koh Tao Island, Surat Thani Province, Railey Beach, Krabi Province, and the Patong district of Phuket Province. The median time to develop TD was 9 days (IQR 5-18 days) post-arrival.

Survival analysis of TD and associated risk factors. We determined the differences in possible risk behaviors between people coming from countries with a high incidence of diarrhea compared with those coming from a low-risk continent and found that only hand washing after using the toilet was the only different behavioral risk factor $(P=0.007)$. Eating street food, using tap water, and travel with children and the elderly did not differ between groups of participants.

We performed a survival analysis testing for all factors, including demographic data, diet, behavior, and activities of participants using the log-rank test. We identified factors significantly associated with an increase in TD incidence, namely, age, drinking tap water, and eating street food. We found no increased TD risk associated with other dietary factors or travel activities except arrival from another Southeast Asian country or travel outside Thailand within the duration of follow-up. The factors significantly associated with decreased TD incidence included routinely washing hands after toilet use and travel with children or the elderly (Table 2).

In addition, we tested all the factors mentioned using the univariate Cox-regression analysis and tested all those identified as having a significant association with TD using a multivariate Cox-regression analysis (Table 3). We identified age, drinking tap water, and arrival from Southeast Asia or travel abroad during the follow-up as the factors that were significant in both univariate and multivariate Cox-regression analyses.

\section{DISCUSSION}

This is a clinic-based prospective study about TD conducted among adult international travelers who traveled to Thailand. In this study, most of the participants were from the developed countries, including North America and Europe. Most previous studies have been retrospective in nature. Few clinic-based prospective cohort studies have been conducted among Europeans traveling to developing countries, including Southeast Asia. ${ }^{6,15,16}$ About one-fifth of the participants had TD within 14 days and one-third had TD within 28 days of arrival in this study. 
TRAVELERS' DIARRHEA IN THAILAND

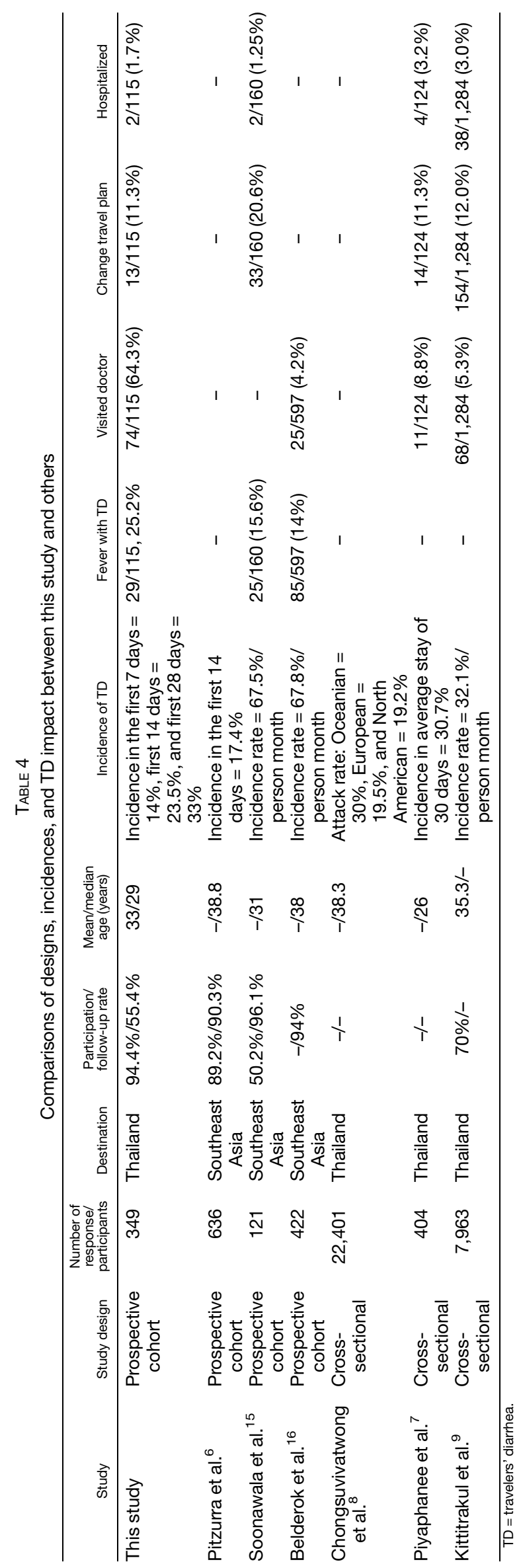


The cumulative incidence of TD during the first 14 days in this study is higher than the incidences found a decade ago. .,6 $^{5}$ However, the TD incidence in this study was lower than that found among travelers to Southeast Asia by clinics in the Netherlands ${ }^{15,16}$ and higher than the incidence demonstrated among adult foreign travelers to Thailand ${ }^{7,9}$ (Table 4). The median duration before TD occurrence in this study was 9 days, and this was longer than that in the study conducted among backpackers in Thailand but shorter than that in a prospective study conducted in Switzerland. ${ }^{6,7}$ The differences in the median time to develop TD may be due to the differences between study populations. In this study, we observed a decrease in the incidence rate of TD starting after the second week.

The impact of TD on travel plan changes was similar to those reported in two other studies, ${ }^{7,9}$ but lower than that in another study. ${ }^{15}$ The hospitalizations due to diarrhea were lower in this study; however, more participants visited physicians because of diarrhea in our study than in others. ${ }^{7,9}$ (Table 4). The mean duration of diarrhea in the study with backpackers was 4 days compared with 2 days in our study. ${ }^{7}$ This difference in impact may be explained by the advice received by the travelers in our study at the travel clinic on enrollment.

In this study, we studied many factors for their association with TD for the first time (allergy to milk, drugs or seafood, routinely washing hands after using the toilet, traveling with children or elderly persons, arrival in Thailand from other Southeast Asian countries, or travel out of Thailand during the follow-up period).

We found no specific types of food associated with the TD incidence, although we looked into factors that have never been studied such as eating insects or eating with bare hands. However, eating street food was found to be significantly associated with the TD incidence, in agreement with findings in other studies. ${ }^{4,9}$ Ice consumption, which had been significantly associated with TD in the past, did not show a significant association with TD in this study. ${ }^{7,9}$ This may be due to the campaign by the Thai government to improve the quality of the ice, as well as improvement in the knowledge among travelers who seek pretravel consultation. Hence, travelers may have avoided consuming ice from unreliable sources. The previous systematic review revealed that personal hygiene precautions, without the direct supervision of an expatriate operating his or her own kitchen, seem to be ineffective in preventing TD. This might be associated with poor food vendor hygiene in most developing countries, which continues to create an insurmountable risk of acquiring TD. ${ }^{17}$

In many past studies, drinking tap water was not associated with the TD incidence. ${ }^{7,9}$ However, it was found to be significantly associated with the incidence of TD in one of the studies, ${ }^{8}$ as well as in this one. Atopy (such as allergic asthma) was associated with TD in a study6; however, we found no associations with allergies.

Other studies, but not the present one, have found associations of handwashing or the use of an alcohol hand cleanser routinely before meals with TD. ${ }^{18,19}$ We found that handwashing after using the toilet was a significant protective factor. However, handwashing after visiting the toilet could serve as a proxy for general hand hygiene. We also found that traveling with children or elderly persons is a TD protective factor, probably because of increased precautions taken by travelers to protect the vulnerable people in their group. This indicates that precautions may reduce the risk of TD if they are actually followed. Travelers arriving from other Southeast Asian countries or traveling outside Thailand during the follow-up period were also associated with a higher risk of TD.

In other studies, people from Australia and Oceania were more susceptible to TD. ${ }^{8,9}$ In this study, the highest followup rate was observed in travelers from those regions (about $10 \%$ more responses than those from participants from Europe and North America). However, this group of participants did not show an association with the incidence of TD in our study.

In other studies, the use of some medications, such as antidepressants and antacids, was found to be associated with an increase in the incidence of TD. ${ }^{6,15}$ Although we tested many groups of medications for associations with TD incidence (antibiotics, antidiarrheal and antimalarial agents, laxatives, antidepressants, and antacids), we found no such associations.

The strength of this study is the prospective collection of data throughout the 4-week follow-up period. The limitations of this study include the design of this study, which does not directly reflect the general population, the possibility that some results may be contaminated as some of the participants traveled outside Thailand. Some data are missing because of the nature of the study design, where data were collected while travelers were traveling.

In conclusion, about one-third of adult foreign travelers experienced travelers' diarrhea within 28 days after arrival in Thailand. We identified factors, including tap water, street food, hand washing, and travel with children or the elderly, associated with the incidence of TD. Although no highly effective TD prevention method exists, the practice of good personal hygiene and the avoidance of food and drinks derived from unsanitary sources are still recommended to reduce the risk of travelers' TD.

Received September 10, 2019. Accepted for publication January 11, 2020.

Published online February 24, 2020.

Note: Supplemental questionnaire appears at www.ajtmh.org.

Acknowledgments: We would like to express our gratitude to the staff of the Hospital for Tropical Diseases, Department of Clinical Tropical Medicine, the Office of Research Services, Faculty of Tropical Medicine, Mahidol University, Thailand, and staff of the Queen Saovabha Memorial Institute, Faculty of Medicine, Chulalongkorn University, Bangkok, Thailand, for their support with data collection.

Financial support: This work was supported by the Faculty of Tropical Medicine, Mahidol University. The funders had no role in study design, data collection and analysis, decision to publish, or preparation of the manuscript.

Authors' addresses: Chollasap Sharma, Department of Clinical Tropical Medicine, Faculty of Tropical Medicine, Mahidol University, Bangkok, Thailand, and Department of Disease Control, Ministry of Public Health, Institute for Urban Disease Control and Prevention, Bangkok, Thailand, E-mail: chollasap.s@gmail.com. Kittiyod Poovorawan, Watcharapong Piyaphanee, Weerapong Phumratanaprapin, Wattana Leowattana, and Polrat Wilairatana, Department of Clinical Tropical Medicine, Faculty of Tropical Medicine, Mahidol University, Bangkok, Thailand, E-mails: kittiyod.poo@mahidol.ac.th, watcharapong.piy@mahidol.ac.th, weerapong.phu@mahidol.ac.th, wattana.leo@mahidol.ac.th, and polrat.wil@mahidol.ac.th. Ngamphol Soonthornworasiri, Department of Tropical Hygiene, Faculty of Tropical Medicine, Mahidol University, Bangkok, Thailand, E-mail: 
ngamphol.soo@mahidol.ac.th. Piyada Angsuwatcharakon, Queen Saovabha Memorial Institute, Faculty of Medicine, Chulalongkorn University, Bangkok, Thailand, E-mail: piyada.angs@gmail.com.

\section{REFERENCES}

1. WHO, 2018. Travel and Health. Available at: http://www.who.int/ topics/travel/en/. Accessed August 18, 2018.

2. Connor BA, 2020. Part 2: traveler's diarrhea. Brunette GW, Nemhauser JB, eds. CDC-Yellow Book 2020: Health Information for International Travel. Atlanta, GA: Oxford University Press, 114-120.

3. UNWTO, 2017. Thailand: Country-specific: Basic indicators (Compendium) 2012-2016. Available at: https://www.eunwto.org/doi/ pdf/10.5555/unwtotfb0764010020132017201812. Accessed March 15, 2018.

4. Steffen R, 2005. Epidemiology of traveler's diarrhea. Clin Infect Dis 41 (Supp/ 8): S536-S540.

5. Steffen R, Hill DR, DuPont HL, 2015. Traveler's diarrhea: a clinical review. JAMA 313: 71-80.

6. Pitzurra R, Steffen R, Tschopp A, Mutsch M, 2010. Diarrhoea in a large prospective cohort of European travellers to resourcelimited destinations. BMC Infect Dis 10: 231.

7. Piyaphanee W, Kusolsuk T, Kittitrakul C, Suttithum W, Ponam T, Wilairatana P, 2011. Incidence and impact of travelers' diarrhea among foreign backpackers in southeast Asia: a result from Khao San road, Bangkok. J Travel Med 18: 109-114.

8. Chongsuvivatwong $V$ et al., 2009. Epidemiology of travelers' diarrhea in Thailand. J Travel Med 16: 179-185.

9. Kittitrakul C, Lawpoolsri S, Kusolsuk T, Olanwijitwong J, Tangkanakul W, Piyaphanee W, 2015. Traveler's diarrhea in foreign travelers in southeast Asia: a cross-sectional survey study in Bangkok, Thailand. Am J Trop Med Hyg 93: 485-490.
10. Hill DR, Ford L, Lalloo DG, 2006. Oral cholera vaccines: use in clinical practice. Lancet Infect Dis 6: 361-373.

11. Ericsson CD, DuPont HL, 1993. Travelers' diarrhea: approaches to prevention and treatment. Clin Infect Dis 16: 616-624.

12. Connor BA, Riddle MS, 2013. Post-infectious sequelae of travelers' diarrhea. J Travel Med 20: 303-312.

13. Tam CC, O'Brien SJ, Rodrigues LC, 2006. Influenza, Campylobacter and Mycoplasma infections, and hospital admissions for GuillainBarre syndrome, England. Emerg Infect Dis 12: 1880-1887.

14. Bodhidatta L, Anuras S, Sornsakrin S, Suksawad U, Serichantalergs O, Srijan A, Sethabutr O, Mason CJ, 2019. Epidemiology and etiology of traveler's diarrhea in Bangkok, Thailand, a case-control study. Trop Dis Travel Med Vaccines 5: 9.

15. Soonawala D, Vlot JA, Visser LG, 2011. Inconvenience due to travelers' diarrhea: a prospective follow-up study. BMC Infect Dis 11: 1-10.

16. Belderok SM, Van den Hoek A, Kint JA, Schim van der Loeff MF, Sonder GJ, 2011. Incidence, risk factors and treatment of diarrhoea among Dutch travellers: reasons not to routinely prescribe antibiotics. BMC Infect Dis 11: 295.

17. Shlim DR, 2005. Looking for evidence that personal hygiene precautions prevent travelers' diarrhea. Clin Infect Dis 41 (Suppl 8): S531-S535.

18. Henriey D, Delmont J, Gautret P, 2014. Does the use of alcoholbased hand gel sanitizer reduce travelers' diarrhea and gastrointestinal upset?: a preliminary survey. Travel Med Infect Dis 12: 494-498.

19. Dreyfuss MS, 2009. Is norovirus a foodborne or pandemic pathogen? An analysis of the transmission of norovirus-associated gastroenteritis and the roles of food and food handlers. Foodborne Pathog Dis 6: 1219-1228. 